\title{
Temperatura de polimerização da resina acrílica odontológica na medula espinhal de ratos Wistar
}

\author{
[Polymerization temperature of dental acrylic resin in the spinal cord of rats] \\ R.P. Santos ${ }^{1}$, A. Mazzanti ${ }^{2 *}$, D.V. Beckmann ${ }^{1}$, G. Aiello ${ }^{1}$, J.S. Brum ${ }^{1}$, P.T. Leme Junior ${ }^{1}$, \\ A. Rippingler ${ }^{3}$, D.P. Neto ${ }^{3}$, T.F. Miranda ${ }^{3}$ \\ ${ }^{1}$ Aluno de pós-graduação - Universidade Federal de Santa Maria - Santa Maria, RS \\ ${ }^{2}$ Universidade Federal de Santa Maria - Santa Maria, RS \\ ${ }^{3}$ Aluno de graduação - Universidade Federal de Santa Maria - Santa Maria, RS \\ RESUMO
}

\begin{abstract}
Objetivou-se investigar se a temperatura de polimerização da resina acrílica odontológica ocasiona sinais neurológicos e alteração histológica na medula espinhal de ratos. Foram utilizados 48 ratos, Wistar, distribuídos em dois grupos denominados GI ou cimento ósseo (controle positivo) e GII ou resina acrílica odontológica. Cada grupo foi redistribuído em seis subgrupos, de acordo com a quantidade do composto, o tempo de pós-operatório e o local de aferição da temperatura. O cimento ósseo ou a resina acrílica odontológica foram moldados e colocados sobre as lâminas ósseas dorsais e os processos espinhosos das vértebras L1 e L2. A temperatura de polimerização do composto foi aferida a cada 10 segundos. A temperatura máxima de polimerização e a diferença entre a temperatura externa e a interna ao canal vertebral foram maiores nos subgrupos que receberam 10 gramas. Não foi observada alteração neurológica em nenhum dos animais deste estudo. Na análise histológica, foi observada reação inflamatória de intensidade variável na meninge e no parênquima medular. Pode-se concluir que a temperatura de polimerização da resina acrílica odontológica nas quantidades de um e 10 gramas provoca alterações histológicas na meninge e no parênquima medular, sem ocasionar sinais neurológicos em ratos.
\end{abstract}

Palavras-chave: rato, neurologia, cirurgia, reação inflamatória

\begin{abstract}
The purpose of this study was to evaluate if the polymerization temperature of the dental acrylic leads to neurological signs and histological changes in the rat spinal cord. Forty eight rats were distributed in two groups: GI or bone cement (positive control) and GII or dental acrylic resin. Each group was redistributed in six subgroups according to compound quantity, postoperative period and location of temperature measurement. The bone cement or dental acrylic resin were molded and positioned on the bony lamina and the dorsal spinous processes of vertebrae L1 and L2. The polimerization temperature was measured every 10 seconds. The polymerization maximum temperature and the temperature difference between internal and external spinal canals were superior in subgroups that received 10 grams. There were no neurological deficits in any animal of this study. The histological analysis showed intensity variation of inflammatory reaction in the meninges and spinal cord parenchyma. The results demonstrate that the polymerization temperature of dental acrylic resin in quantity of 1 or 10 grams causes histological changes in the spinal cord parenchyma and meninges, without neurological deficits in rats.
\end{abstract}

Keywords: rat, neurology, surgery, inflammation

Recebido em 19 de março de 2011

Aceito em 30 de maio de 2012

*Autor para correspondência (corresponding author)

E-mail: alexamazza@yahoo.com.br 


\section{INTRODUÇÃO}

As fraturas e as luxações vertebrais ocorrem frequentemente em pequenos animais, e o tratamento para estas afecções pode ser clínico ou cirúrgico (Sturges e Lecouteur, 2007). O tratamento cirúrgico é o mais eficaz, pois proporciona o realinhamento e a estabilização precisa das vértebras acometidas (Sharp e Wheeler, 2005), estando sempre indicado quando ocorrer instabilidade vertebral e/ou compressão medular (Young, 1993). Para esta estabilização, são propostas várias técnicas, porém a combinação de pinos de Steimann e cimento ósseo é a técnica preferida em cães e gatos por fornecer uma adequada estabilização e resistência, contribuindo para a cicatrização das fraturas e luxações vertebrais (Sharp e Wheeler, 2005; Sturges e Lecouter, 2007).

O cimento ósseo ortopédico é utilizado como biomaterial desde 1930 (Wiltse et al., 1957), principalmente para fixação de próteses, correção de defeitos ósseos e estabilização das fraturas vertebrais (Stanczkyk e Rietbergen, 2004). O cimento ósseo é constituído por um polímero na forma de pó e um monômero líquido à base de metacrilato de metila, unidos pelo processo de polimerização. Esta polimerização ocorre por meio de uma reação exotérmica, que libera grande quantidade de calor, com as temperaturas variando de $40^{\circ}$ a $110^{\circ} \mathrm{C}$ (Dunne e Orr, 2001). A alta temperatura pode provocar necrose térmica na superfície óssea em contato, o que é considerado um problema durante o processo de polimerização (Dipisa et al., 1976). Wang et al. (1984) analisaram o comportamento do cimento ósseo quando empregado sobre a medula espinhal de cães, não encontrando alterações histológicas e neurológicas.

Alguns estudos foram realizados substituindo o cimento ósseo ortopédico por outros biomateriais, como, por exemplo, a resina acrílica odontológica autopolimerizável (Rahal et al., 1996; Gioso et al., 2001; Goelzer et al., 2003). Assim como o cimento ósseo, a resina acrílica odontológica autopolimerizável também é formada à base de polimetilmetacrilato. Os vários tipos de resina acrílica à base de polimetilmetacrilato são amplamente utilizados em odontologia, tendo inúmeras aplicações, como na confecção de aparelhos ortodônticos removíveis e nas bases para próteses orais odontológicas.

Por ser formada à base de polimetilmetacrilato semelhante ao cimento ósseo, de fácil obtenção e custo reduzido em alguns hospitais e clínicas veterinárias, a resina acrílica odontológica autopolimerizável tem sido utilizada no auxílio da estabilização da coluna vertebral. No Hospital Veterinário da instituição, os cães e gatos que receberam o composto não desenvolveram complicações no pós-operatório, como rejeição e piora nos sinais neurológicos. Mesmo conhecendo-se os resultados clínicos satisfatórios da resina acrílica odontológica na coluna vertebral de cães e gatos, pouco se sabe sobre o comportamento desse composto, a temperatura de polimerização e a influência nos tecidos nervosos.

Diante disso e do fato de não se encontrar na literatura consultada estudo sobre o emprego da resina acrílica odontológica na coluna vertebral, o objetivo deste experimento foi investigar se a temperatura de polimerização da resina acrílica odontológica ocasiona sinais neurológicos e alteração histológica na medula espinhal de ratos.

\section{MATERIAL E MÉTODOS}

Foram utilizados 48 ratos Wistar, machos, com massa corporal variando entre 300 e $400 \mathrm{~g}$, provenientes do Biotério Central da instituição. Os animais foram distribuídos, ao acaso, em dois grupos denominados GI ou cimento ósseo (controle positivo) e GII ou resina acrílica odontológica. Cada grupo foi redistribuído em seis subgrupos, de acordo com a quantidade do composto (um ou 10 gramas), o tempo de pósoperatório ( 24 e 72 horas) e o local de aferição da temperatura (externa e interna ao canal vertebral) (Tab. 1). 
Tabela 1. Temperatura de polimerização da resina acrílica odontológica na medula espinhal de ratos. Distribuição dos grupos cimento ósseo (GI) e resina acrílica odontológica (GII), de acordo com a quantidade de composto, o período de avaliação neurológica e o tempo de pós-operatório

\begin{tabular}{|c|c|c|c|c|}
\hline Grupo & Subgrupo & $\begin{array}{l}\text { Quantidade do } \\
\text { composto (gramas) }\end{array}$ & $\begin{array}{l}\text { Avaliação neurológica } \\
\text { (horas) }\end{array}$ & $\begin{array}{c}\text { Pós-operatório } \\
\text { (horas) }\end{array}$ \\
\hline \multirow{6}{*}{$\begin{array}{c}\text { (GI) } \\
\text { Cimento ósseo }\end{array}$} & $\mathrm{GI}_{1}$ & 1 & 24 & 24 \\
\hline & $\mathrm{GI}_{2}$ & 1 & 24,48 e 72 & 72 \\
\hline & $\mathrm{GI}_{3}$ & 10 & 24 & 24 \\
\hline & $\mathrm{GI}_{4}$ & 10 & 24,48 e 72 & 72 \\
\hline & $\mathrm{GI}_{5}$ & 1 & & $\begin{array}{l}\text { Eutanásia no } \\
\text { transoperatório }\end{array}$ \\
\hline & $\mathrm{GI}_{6}$ & 10 & & $\begin{array}{l}\text { Eutanásia no } \\
\text { transoperatório }\end{array}$ \\
\hline \multirow{6}{*}{$\begin{array}{c}\text { (GII) } \\
\text { Resina acrílica }\end{array}$} & $\mathrm{GII}_{1}$ & 1 & 24 & 24 \\
\hline & $\mathrm{GII}_{2}$ & 1 & 24,48 e 72 & 72 \\
\hline & $\mathrm{GII}_{3}$ & 10 & 24 & 24 \\
\hline & $\mathrm{GII}_{4}$ & 10 & 24,48 e 72 & 72 \\
\hline & $\mathrm{GII}_{5}$ & 1 & & $\begin{array}{l}\text { Eutanásia no } \\
\text { transoperatório }\end{array}$ \\
\hline & $\mathrm{GII}_{6}$ & 10 & & $\begin{array}{l}\text { Eutanásia no } \\
\text { transoperatório }\end{array}$ \\
\hline
\end{tabular}

Os ratos foram mantidos em gaiolas forradas com maravalha, em temperatura ambiente controlada $\left(24^{\circ} \pm 2^{\circ} \mathrm{C}\right)$ e com um ciclo claro/escuro de 12 x 12 horas, alimentados com ração comercial e água ad libitum. Os animais foram identificados, pesados e numerados por meio de um código nas caudas. Este trabalho seguiu as normas de experimentação segundo o Comitê de Ética em Pesquisa da instituição e aprovado conforme processo administrativo número 24087.011222/2010-49.

Para a realização do procedimento cirúrgico, os ratos receberam antibiótico profilático com ampicilina sódica $(20 \mathrm{mg} / \mathrm{kg})$ por via intraperitoneal (IP), 30 minutos antes do início do procedimento. Foi realizada medicação préanestésica com cloridrato de tramadol $(2 \mathrm{mg} / \mathrm{kg})$, por via subcutânea (SC), e manutenção anestésica com halotano administrado por meio de máscara em sistema semiaberto. A temperatura ambiente foi mantida em $23^{\circ} \mathrm{C}(+/-$ $1^{\circ} \mathrm{C}$ ) durante a realização do ato cirúrgico.

Após a realização da tricotomia e em plano anestésico, os ratos foram posicionados em decúbito external sobre a mesa cirúrgica, que foi confeccionada com duas bases de polietileno, separadas por quatro hastes de alumínio, o que permitia o encaixe de uma bolsa com água aquecida a aproximadamente $80^{\circ} \mathrm{C}$ entre as chapas (Torres, 2008). A mesa cirúrgica foi utilizada para evitar a hipotermia dos animais durante o procedimento anestésico-cirúrgico. A antissepsia da pele foi realizada com álcool-iodoálcool.

Realizou-se a incisão da pele e do tecido subcutâneo entre a décima primeira vértebra torácica e a terceira vértebra lombar. Os músculos epaxiais foram incisados, rebatidos com o elevador de periósteo e afastados com o auxílio do afastador autoestático de Weitlaner, mantendo expostas as lâminas ósseas dorsais e os processos espinhosos de L1 e L2.

O cimento ósseo (BAUMER OSTEO-CLASS, Rua 24 de Outubro, no 1921 - Porto Alegre, RS) e a resina acrílica odontológica (VIP FLASH Rua Carlos Tassoni, n4521- Pirassununga - São Paulo, SP) foram preparados manualmente nas proporções 2:1 (10 gramas de pó e cinco mililitros de líquido), em uma cuba de aço inox, com auxílio de uma espátula de madeira, previamente esterilizadas. Ao atingir a fase de modelagem, o composto foi pesado em uma balança de precisão, em recipiente estéril. Foi utilizado para os subgrupos $\mathrm{GI}_{1}, \mathrm{GI}_{2}$ e $\mathrm{GI}_{5} \mathrm{um}$ grama, e para os subgrupos $\mathrm{GI}_{3}, \mathrm{GI}_{4}$ e $\mathrm{GI}_{6} 10$ gramas de cimento ósseo. As mesmas quantidades foram definidas com a resina acrílica odontológica e distribuídas, ou seja, um grama para os subgrupos $\mathrm{GII}_{1}, \mathrm{GII}_{2}$ e $\mathrm{GII}_{5}$ e 10 gramas para $\mathrm{GII}_{3}, \mathrm{GII}_{4}$ e $\mathrm{GII}_{6}$. A quantidade de um grama de cimento ósseo ou resina acrílica definida neste estudo foi estabelecida de acordo com a rotina 
cirúrgica, na qual é utilizado, aproximadamente, entre $0,3 \%$ e $0,4 \%$ do peso do animal. Também foi objetivo desta pesquisa investigar o efeito da temperatura de polimerização se utilizada 10 vezes mais a quantidade normalmente empregada, a fim de avaliar se a grande quantidade de material influenciaria no aumento de temperatura e nas lesões aos tecidos nervosos.

O cimento ósseo ou a resina acrílica odontológica foram moldados e colocados sobre as lâminas ósseas dorsais e os processos espinhosos das vértebras L1 e L2. Em seguida, foi feita a aferição da temperatura do composto com auxílio de termopares tipo $\mathrm{K}$ acoplados a um termômetro digital portátil (TERMOSEG, Rua Francisco Fett, 537 - São Paulo, SP). Um dos termopares foi posicionado longitudinalmente entre o composto (cimento ósseo ou a resina acrílica odontológica) e as lâminas ósseas dorsais das vértebras L1 e L2. Após o término das aferições, que foram anotadas em protocolos específicos, nos animais dos subgrupos $\mathrm{GI}_{1}, \mathrm{GI}_{2}, \mathrm{GI}_{3}, \mathrm{GI}_{4}$ (cimento ósseo) e $\mathrm{GII}_{1}, \quad \mathrm{GII}_{2}, \quad \mathrm{GII}_{3}, \mathrm{GII}_{4}$ (resina acrílica odontológica), o composto foi removido, e foi realizada a sutura das camadas musculares, do tecido subcutâneo e da pele com pontos contínuos simples, empregando-se fio mononáilon $\mathrm{n}^{\circ} 4-0$. Já nos subgrupos $\mathrm{GI}_{5}, \mathrm{GI}_{6}$ (cimento ósseo) e $\mathrm{GII}_{5}, \mathrm{GII}_{6}$ (resina acrílica odontológica), foi realizada uma laminectomia dorsal modificada sobre as vértebras L3-L4, com o auxílio de uma perfuratriz de baixa rotação, e um segundo termopar tipo $\mathrm{K}$ foi inserido no interior do canal vertebral dorsal à medula espinhal, permanecendo no mesmo ponto do primeiro termopar situado fora do canal vertebral. Desta maneira, foi possível avaliar simultaneamente a temperatura externa e a interna do canal vertebral. Os animais destes subgrupos $\left(\mathrm{GI}_{5}, \mathrm{GI}_{6}\right.$ e $\left.\mathrm{GII}_{5}, \mathrm{GII}_{6}\right)$ foram submetidos à eutanásia no transoperatório, previamente à introdução do segundo termopar no interior do canal vertebral.

A variação da temperatura de polimerização do cimento ósseo ou da resina acrílica foi verificada a partir da sua colocação sobre as vértebras L1L2 (T0), sendo aferida a cada 10 segundos, durante todo o período de aquecimento e resfriamento. O término da aferição ocorreu quando a temperatura do composto atingiu o equilíbrio térmico com a temperatura retal do animal, tendo em vista que esta temperatura já não seria mais deletéria para os tecidos em contato com os compostos.

Após o procedimento cirúrgico, os animais foram colocados em uma caixa e aquecidos a $37^{\circ} \mathrm{C}$ por uma luz incandescente de 100 watts a uma distância mínima de $50 \mathrm{~cm}$ durante, aproximadamente, 10 minutos e devolvidos para as suas caixas originais. Os ratos receberam analgesia pós-operatória realizada com cloridrato de tramadol $(2 \mathrm{mg} / \mathrm{kg}, \mathrm{SC})$ a cada oito horas por 24 horas e meloxicam $(10 \mathrm{mg} / \mathrm{kg} \mathrm{SC})$ uma vez ao dia, durante três dias.

Os subgrupos $\mathrm{GI}_{1}, \mathrm{GI}_{3}, \mathrm{GII}_{1}, \mathrm{GII}_{3}$ foram submetidos à eutanásia decorridas 24 horas, e os $\mathrm{GI}_{2}, \mathrm{GI}_{4}, \mathrm{GII}_{2}, \mathrm{GII}_{4} 72$ horas do procedimento cirúrgico. A eutanásia foi realizada com inalação de halotano no interior de um recipiente, seguindo a legislação em vigor e os preceitos do Colégio Brasileiro de Experimentação Animal (COBEA). O segmento envolvendo as vértebras L1-L2 foi removido e fixado em formol a $10 \%$ para análise histológica.

O teste neurológico foi realizado por meio da escala de Basso, Beattie e Bresnahan (BBB) (Basso et al., 1995) e do plano inclinado (Rivlin e Tator, 1977). Estas avaliações foram feitas antes (24 horas) e decorridos os períodos preestabelecidos de pós-operatório. Os resultados foram anotados em protocolos específicos.

Para análise histológica, as vértebras L1 e L2, incluindo a medula espinhal, foram descalcificadas com ácido fórmico, cortadas longitudinalmente e coradas pela técnica de Hematoxilina de Harris e Eosina. As estruturas analisadas foram a meninge e o parênquima medular entre L1 e L2. As alterações encontradas foram classificadas em ausente (não houve a observação de infiltrado inflamatório), leve (infiltração restrita a uma pequena área, sem invasão profunda e formada por uma pequena quantidade de células), moderado (os infiltrados se caracterizavam por um ou mais focos, com um significativo número de células, porém substituindo pequena parte do parênquima) e grave (as lesões abrangiam uma área focalmente extensa, ocupando substancialmente o parênquima, aliada a uma marcada quantidade de células inflamatórias). 
Os dados obtidos das análises histológicas e dos testes neurológicos foram avaliados estatisticamente, com auxílio de testes não paramétricos, teste Kruskal-Wallis e teste $\mathrm{t}$, com nível de significância de $5 \%$.

\section{RESULTADOS}

A temperatura média máxima de polimerização do cimento ósseo (GI) foi de $53,5^{\circ} \mathrm{C}$ e para resina acrílica odontológica (GII) de $56,7^{\circ} \mathrm{C}$, quando os compostos pesavam de um grama, e de $68,6^{\circ} \mathrm{C}$ (GI) e $59,1^{\circ} \mathrm{C}$ (GII) para os que pesavam 10 gramas, havendo diferença significativa $(\mathrm{P}<0,05)$ entre as quantidades testadas (Fig. 1A e 1B). Não foi observada diferença significativa quanto à quantidade de compostos.

Quanto aos valores de temperaturas máximas de polimerização (externo e interno) ao canal vertebral, foi verificado, em média, nos animais do subgrupo $\mathrm{GI}_{5}\left(48,2^{\circ} \mathrm{C}\right.$ e $\left.42,8{ }^{\circ} \mathrm{C}\right)$, no $\mathrm{GI}_{6}$ $\left(61,9^{\circ} \mathrm{C} \mathrm{e} 48,7^{\circ} \mathrm{C}\right)$, no $\mathrm{GII}_{5}\left(55,2^{\circ} \mathrm{C}\right.$ e $\left.42,6^{\circ} \mathrm{C}\right)$ e no $\mathrm{GII}_{6} \quad\left(64^{\circ} \mathrm{C}\right.$ e $\left.46,2^{\circ} \mathrm{C}\right)$, não apresentando diferenças significativas entre os subgrupos com a mesma quantidade do composto, ou seja, $\mathrm{GI}_{5} \mathrm{e}$ $\mathrm{GII}_{5}$ (um grama) e $\mathrm{GI}_{6}$ e $\mathrm{GII}_{6}$ (10 gramas). Ao se comparar, porém, a temperatura entre subgrupos com quantidades diferentes, pôde-se verificar diferença significativa $(\mathrm{P}<0,05)$ entre eles, ou seja, as temperaturas externas e internas ao canal vertebral foram maiores nos animais que receberam 10 gramas do composto (Fig. 1C e 1D). Mesmo não havendo diferença significativa, o grupo GI (cimento ósseo) apresentou uma diferença, em média, de $5,4^{\circ} \mathrm{C}$ entre a temperatura externa e a interna ao canal vertebral e do GII (resina acrílica odontológica) de $12,6^{\circ} \mathrm{C}$, ambos utilizando um grama do composto. Já ao empregar 10 gramas, o grupo GI teve, em média, uma diferença de $13,2^{\circ} \mathrm{C}$ e do GII $17,8^{\circ} \mathrm{C}$.

$\mathrm{Na}$ análise histológica da meninge, dos grupos GI e GII, foram observadas alterações vasculares, caracterizadas por congestão de vasos sanguíneos (Fig. 2A), acompanhada por edema intersticial e ocasionalmente hemorragia. A reação inflamatória foi de intensidade leve em $67 \%$ do $\mathrm{GI}_{1}$, em todos os animais do grupo $\mathrm{GI}_{3}$, em $66 \%$ do $\mathrm{GI}_{4}$, em $80 \%$ do $\mathrm{GII}_{1}$ e do $\mathrm{GII}_{3}$, em $60 \%$ do $\mathrm{GII}_{2}$ e em $40 \%$ do $\mathrm{GII}_{4}$; moderada em todos os animais do subgrupo $\mathrm{GI}_{2}$ e em $40 \%$ do $\mathrm{GII}_{4}$; severa em $33 \%$ do $\mathrm{GI}_{4}$ e em $20 \%$ do $\mathrm{GII}_{4}$. As alterações histológicas foram significativas
$(\mathrm{P}<0,05)$ na meninge de ratos que receberam 10 gramas (subgrupos $\mathrm{GI}_{3}, \mathrm{GI}_{4}, \mathrm{GII}_{3}, \mathrm{GII}_{4}$ ).

As lesões histológicas do parênquima medular caracterizaram-se principalmente por uma infiltração restrita a uma pequena área, sem invasão profunda e com uma pequena quantidade de células (Fig. 2B). A alteração foi de intensidade leve em $67 \%$ do $\mathrm{GI}_{4}$, em $20 \%$ do $\mathrm{GII}_{1}$ e do $\mathrm{GII}_{2}$, em $60 \%$ do $\mathrm{GII}_{4}$; grave em $20 \%$ do $\mathrm{GII}_{4}$; ausente em $33 \%$ do $\mathrm{GI}_{4}$, em $80 \%$ do $\mathrm{GII}_{1}$ e do $\mathrm{GII}_{2}$, em $20 \%$ do $\mathrm{GII}_{4}$ e em todos os animais dos subgrupos $\mathrm{GI}_{1}, \mathrm{GI}_{2}, \mathrm{GI}_{3}, \mathrm{GII}_{3}$. Os animais dos subgrupos com tempo de pósoperatório de 72 horas apresentaram alterações histológicas mais significativas $(\mathrm{P}<0,05)$ quando comparados aos de 24 horas de pós-operatório.

Nos tecidos nervosos (meninge e parênquima medular) dos ratos dos grupos GI e GII, foram encontrados infiltrados celulares inflamatórios perivasculares com predominância de neutrófilos. Por outro lado, nos subgrupos resina acrílica odontológica, houve também um predomínio de linfócitos.

A média do tempo de polimerização (aquecimento e resfriamento) do cimento ósseo foi de 723 segundos no $\mathrm{GI}_{1}$ e no $\mathrm{GI}_{2}$, e 1861 segundos no $\mathrm{GI}_{3}$ e no $\mathrm{GI}_{4}$. Já nos subgrupos da resina acrílica odontológica, foi no $\mathrm{GII}_{1}$ e no $\mathrm{GII}_{2}$ de 813 segundos, e no $\mathrm{GII}_{3}$ e $\mathrm{GII}_{4}$ de 2027 segundos (Fig. 2A e Fig. 2B). Nos subgrupos $\mathrm{GI}_{1}$ e $\mathrm{GI}_{2}$, o tempo de permanência da temperatura acima de $42^{\circ} \mathrm{C}$ foi de 157 segundos, nos subgrupos $\mathrm{GI}_{3}$ e GI 4 de 1530 segundos, nos $\mathrm{GII}_{1} \mathrm{e}$ $\mathrm{GII}_{2}$ de 186 segundos e de 1346 segundos nos subgrupos $\mathrm{GII}_{3}$ e $\mathrm{GII}_{4}$.

Nenhum dos grupos estudados apresentou alterações no exame neurológico.

\section{DISCUSSÃO}

A colocação do cimento ósseo e da resina acrílica odontológica sobre as lâminas ósseas vertebrais e os processos espinhosos, com a finalidade de avaliar o efeito da temperatura de polimerização sobre a medula espinhal, foi semelhante ao procedimento empregado na rotina cirúrgica, visto que estes compostos são distribuídos nessas regiões para fixar os pinos e estabilizar a fratura ou a luxação vertebral. 


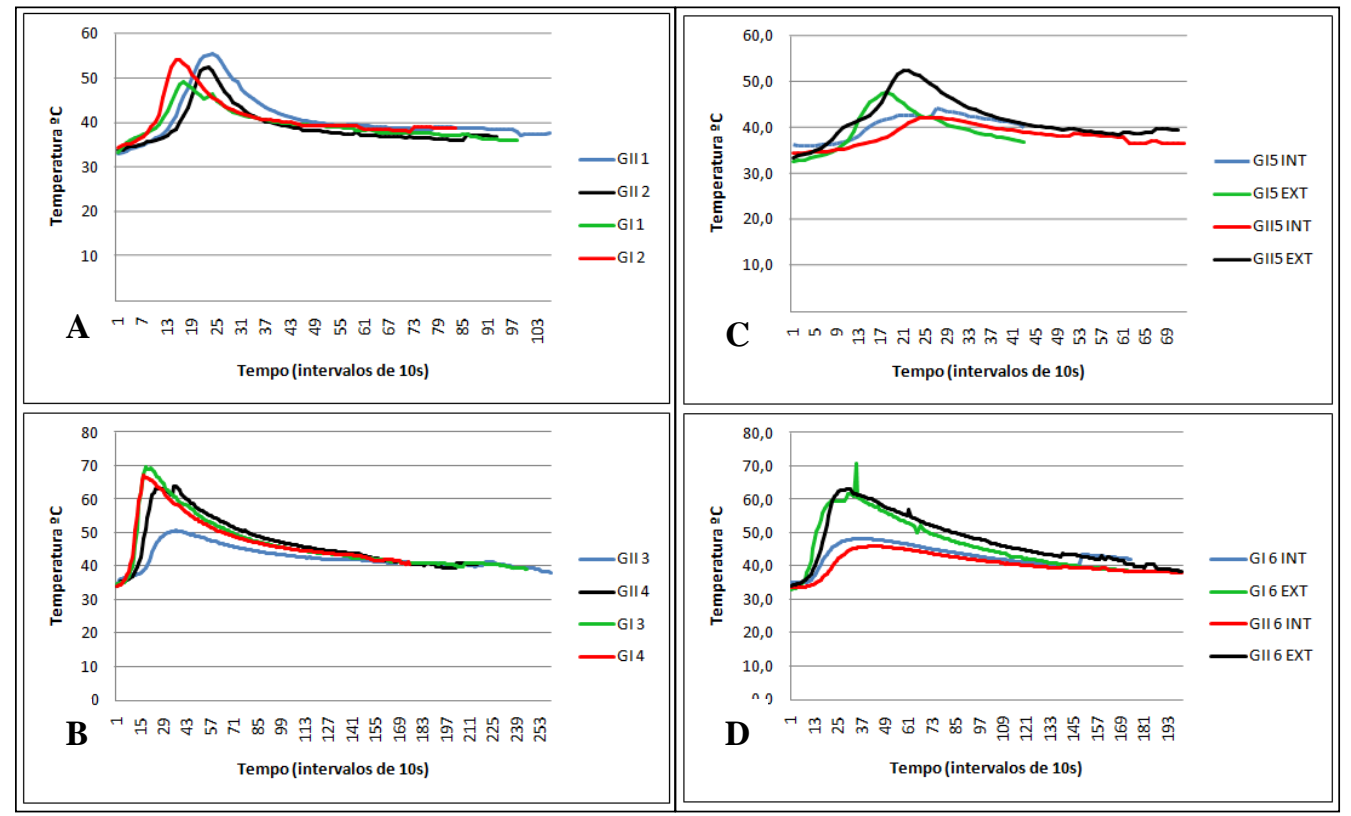

Figura 1. Tempo da temperatura de polimerização da resina acrílica odontológica na medula espinhal de ratos. (A) Curvas de polimerização dos subgrupos $\mathrm{GI}_{1}, \mathrm{GI}_{2}, \mathrm{GII}_{1}, \mathrm{GII}_{2}$. (B) Curvas de polimerização dos subgrupos $\mathrm{GI}_{3}, \mathrm{GI}_{4}, \mathrm{GII}_{3}, \mathrm{GI}_{4}$. (C) Curvas de polimerização dos subgrupos $\mathrm{GI}_{5}$ e $\mathrm{GII}_{5}$ (EXT [temperaturas externas ao canal medular], INT [temperaturas internas ao canal vertebral]). (D) Curvas de polimerização dos subgrupos $\mathrm{GI}_{6}$ e $\mathrm{GII}_{6}$ (EXT [temperaturas externas ao canal medular], INT [temperaturas internas ao canal vertebral]).

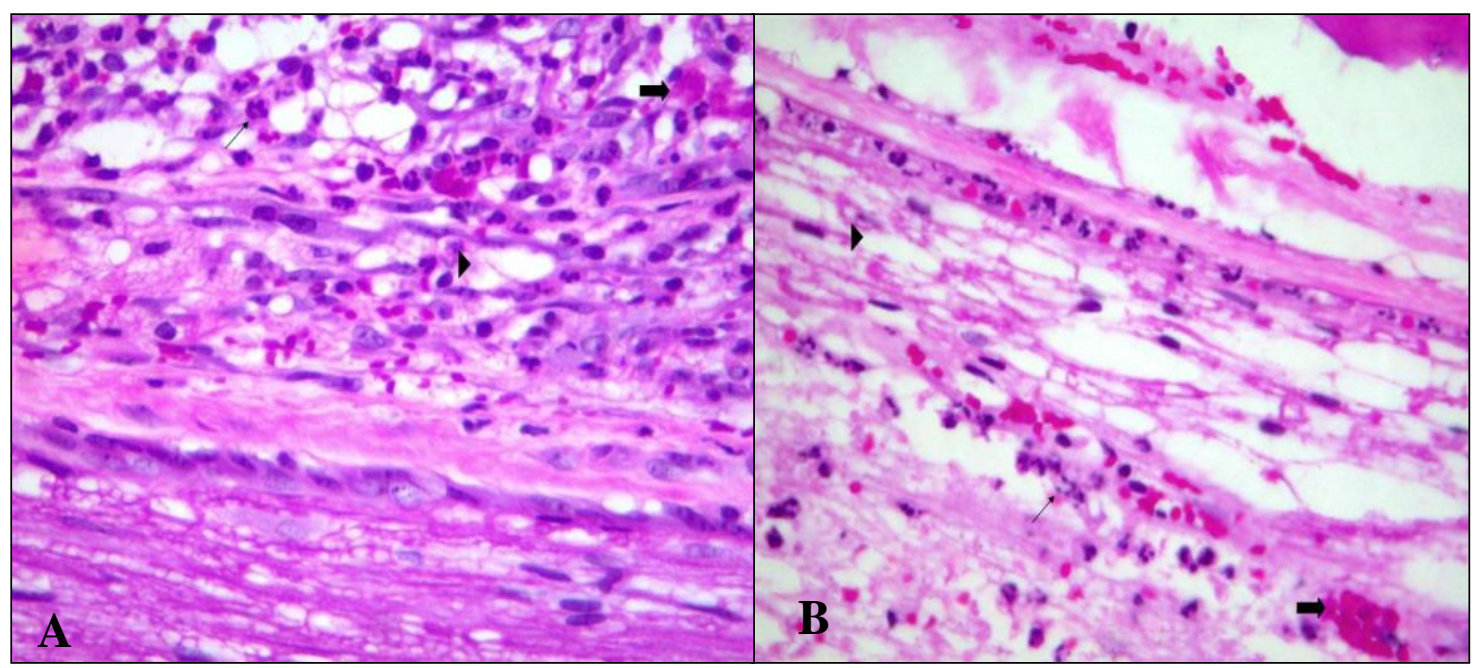

Figura 2. Rato. Fotomicrografia mostrando o efeito da temperatura de polimerização da resina acrílica odontológica na medula espinhal na avaliação de 72 horas. (A) Lesão histológica observada na meninge (GII). Verifica-se um espessamento da região devido a acentuado infiltrado inflamatório constituído predominantemente de neutrófilos (seta fina) e uma menor quantidade de linfócitos. Edema moderado (ponta de seta) e congestão leve de vasos sanguíneos (seta espessa). HE. (B) Lesão histológica observada na medula espinhal (GII). Área focalmente extensa de substituição de parte da substância branca por acentuado infiltrado inflamatório constituído predominantemente de neutrófilos (seta fina). Edema acentuado (ponta de seta) e congestão moderada de vasos sanguíneos (seta espessa). HE. 
A quantidade de material foi estabelecida proporcionalmente, de acordo com a utilizada na rotina clínica. Blass e Seim (1984) utilizaram em cães com mais de $15 \mathrm{~kg}$ aproximadamente $40 \mathrm{~g}$ de cimento ósseo, o que correspondia a $0,26 \%$ do peso corporal.

A temperatura média máxima de polimerização foi proporcional à quantidade de cimento ósseo e de resina acrílica aplicados, corroborando com os achados de Revie et al. (1994), nos quais eles também verificaram que a quantidade de polimetilmetacrilato influenciou na variação da temperatura. Não foi observada diferença significativa quanto à quantidade de compostos nos grupos GI e GII, provavelmente porque ambos são formados de polimetilmetacrilato (Moraes et al., 2003; Jaeblon, 2010).

A menor temperatura encontrada na parte interna do canal vertebral pode ser explicada pela presença das estruturas de proteção da medula espinhal representada, principalmente, pela lâmina óssea dorsal e pelo processo espinhoso vertebral, que contribuíram para a dissipação e provável atenuação dos efeitos do calor na medula espinhal. A maior diferença de temperatura externa e interna da resina acrílica pode sugerir uma tendência de a resina acrílica odontológica apresentar maior perda de calor para o meio quando comparada ao cimento ósseo.

Os resultados histológicos revelaram que, mesmo na presença das estruturas que protegem a medula espinhal, a temperatura encontrada no interior do canal vertebral ocasionou diferentes intensidades de alterações histológicas, sendo mais significativas na meninge de ratos que receberam 10 gramas (subgrupos $\mathrm{GI}_{3}, \mathrm{GI}_{4}, \mathrm{GII}_{3}$, $\mathrm{GII}_{4}$ ), por permaneceram expostos a maiores temperaturas de polimerização, fazendo com que o dano tecidual fosse mais intenso e de maior expansão. Além de a quantidade do composto influenciar nas modificações histológicas, o tempo de avaliação de até $72 \mathrm{~h}$ após a exposição ao composto pode ter contribuído para o aparecimento das alterações no parênquima medular.

Os infiltrados celulares inflamatórios perivasculares com predominância de neutrófilos encontrados no parênquima medular dos grupos GI e GII provavelmente ocorreram pelas altas temperaturas de polimerização dos compostos, semelhante aos achados de Jones et al. (2000), que observaram um predomínio deste tipo celular em lesões térmicas, mesmo na ausência de infecções secundárias. $\mathrm{O}$ predomínio de linfócitos, geralmente, está presente nas reações alérgicas tardias, e foi observado nos dos subgrupos resina acrílica odontológica. De acordo com Stanczyk e Rietbergen (2004), a resina acrílica odontológica autopolimerizável libera uma quantidade maior de monômeros residuais quando comparados a outros tipos de polímeros, o que justificaria a reação alérgica e a presença de linfócitos. Hochman e Zalkind (1997) e Morais et al. (2007) citaram que os monômeros provocam reações locais devido a sua toxicidade e que tal fato pode justificar a reação inflamatória produzida nas meninges e no parênquima medular. Além disso, a mistura manual dos componentes como foi realizada neste trabalho, provavelmente, contribuiu para a maior liberação de monômeros residuais quando esse método foi comparado aos outros, como o vácuo e a centrifugação, conforme estudos realizados por Bettencourt et al. (2001).

Pode-se perceber que, nos subgrupos em que foi utilizada maior quantidade de composto (10 gramas), o tempo entre o início e o fim do aquecimento até se igualar à temperatura retal foi maior, resultando em maiores alterações histológicas na meninge e no parênquima medular. Foi verificado por Field e Morris (1983) que os danos celulares in vitro ou in vivo sofrem uma transição quando submetidos a temperaturas na faixa de $42-43^{\circ} \mathrm{C}$. Os ratos dos subgrupos com maior quantidade de composto ficaram mais expostos a temperaturas acima de $42^{\circ} \mathrm{C}$, faixa considerada prejudicial, o que justificaria a diferença estatística da reação inflamatória dos tecidos nervosos em relação às quantidades dos compostos recebidos.

As altas temperaturas de polimerização encontradas nos compostos (cimento ósseo e resina acrílica odontológica) foram suficientes para provocar alterações histológicas na meninge e, em menor grau, no parênquima medular, sem, portanto, ocasionar deficiências neurológicas. Nenhum dos grupos estudados apresentou alterações no exame neurológico. Os mesmos achados histológicos provavelmente seriam encontrados em cães e gatos da rotina cirúrgica ao se empregar a resina acrílica odontológica 
para estabilização da coluna vertebral. Pelos resultados encontrados neste estudo, tanto o cimento ósseo como a resina acrílica odontológica podem ser indicados para estabilização de fraturas ou luxações vertebrais. Embora não fossem observados sinais clínicos e histológicos de infecção, outros estudos deverão ser realizados com a finalidade de avaliar o comportamento da resina acrílica odontológica quanto à presença de infecção nos tecidos.

\section{CONCLUSÕES}

A temperatura de polimerização da resina acrílica odontológica nas quantidades de um e 10 gramas ocasiona alterações histológicas na meninge e no parênquima medular, sem provocar sinais neurológicos em ratos.

\section{REFERÊNCIAS}

BASSO, D.M.; BEATTIE, M.S.; BRESNAHAN, J.C. A sensitive and reliable locomotor rating scale for open field testing in rats. J. Neurotr., v.12, p.1-21, 1995.

BETTENCOURT, A.; CALADO, A.; AMARAL; J. et al. The influence of vacuum mixing on methylmethacrylate liberation from acrylic cement powder. Int. J. Pharmac., v.219, p.89-93, 2001.

BLASS, C.E.; SEIM III, H.B. Spinal fixation in dogs using steinmann pins and methylmethacrylate. Vet. Surg., v.13, p.203-210, 1984.

DIPISA, J.A.; SIH, G.S.; BERMAN, A. The temperature problema the bone-acrylic cement interface of the total hip replacement. Clin. Orthop., v.121, p.95-98, 1976.

DUNNE, N.J.; ORR, J.F. Thermal characteristics of curing acrylic bone cement. ITBM-RBM, v.22, n.2, p.88-97. 2001.

FIELD, S.B.; MORRIS, C.C. The relationship between heating time and temperature: its relevance to clinical hyperthermia. Radioth. Oncol., v.1, p.179-186, 1983.

GIOSO, M.A.; VIANNA, R.S.; VENTURINI, M.A.F.A. et al. Análise clínica e histológica da utilização da resina acrílica autopolimerizável nas fraturas de mandíbula e maxila e separação da sínfise mentoniana em cães e gatos. Cienc. Rural, v.31, p.291-298, 2001.

GOELZER, L.P.; RAISER, A.G.; GAIGA, L.H. et al. Acrílico auto-polimerizável associado ou não a retalho mucoperiostal simples no tratamento de fístula oronasal experimental em cães. Arq. Bras. Med. Vet. Zoot., v.55, p.550-556, 2003.
HOCHMAN, N.; ZALKIND, M. Hypersensitivity to methyl methacrylate: mode of treatment. J. Prost. Dent., v.77, p.93-96, 1997.

JAEBLON, T. Polymethylmethacrylate: properties and contemporary uses in orthopaedics. J. Am. Acad. Orthop. Surg., v.18, p.297-305, 2010.

JONES, T.C.; HUNT, R.D.; KING, N.W. Patologia Veterinária. São Paulo: Manole, 2000. 1415p.

MORAES, M.A.S.; REZENDE, E.J.C.; SOUZA, E.L. A história da resina composta na Odontologia Parte 1. Cienc. Saúde - Rev. Cromg., v.9, p.72-77, 2003.

MORAIS, I.F.A.; MELLO, B.A.; SOUZA, I.A. et al. Polímeros a base de metil metacrilato. Importância em odontologia. Int. J. Dent., v.6, p.63-66, 2007.

RAHAL， S.C.; BRACARENSE， A.P.F.R.L.; MIGLIATI, E.R. et al. Implantação intra-orbital, após a enucleação transpalpebral, de resina acrílica ou pericárdio em coelhos. Cienc. Rural, v.26, p.229-233, 1996.

REVIE, I.C.; WALLCE, M.E.; ORR, J.F. The effects of PMMA thickness on thermal bone necrosis around acetabular sockets. Proc. Inst. Mech. Eng., v.208, p.45-51, 1994.

RIVLIN, A.S.; TATOR, C.H. Objective clinical assessment of motor function after experimental spinal cord injury in the rat. J. Neurosurg., v.47, p.577-581, 1977.

SHARP, N.J.H.; WHEELER, S.J. Small animal spinal disorders: Diagnosis and surgery. 2.ed. Philadelphia: Elsevier Mosby, 2005. 380p.

STANCZKYK, M.; RIETBERGEN, V. Thermal analysis of bone cement polymerization at the cementbone interface. J. Biomech., v.37, p.1803-1810, 2004.

STURGES, B.K.; LECOUTEUR, R.A. Fraturas e luxações vertebrais, In: SLATTER, D. Manual de cirurgia de pequenos animais. 3.ed. São Paulo: Manole, 2007. v.1, cap.83, p.1244-1260.

TORRES, B.B.J. Efeitos do dantrolene sódico em ratos adultos com trauma medular agudo experimental. 2008. 84f. Dissertação (Mestrado em Medicina Veterinária) - Universidade Federal de Minas Gerais, Belo Horizonte.

WANG, G.J.; REGER, S.I.; SHAO, Z.H. et al. Comparative strength of anterior spinal fixation whith bone graft or polymethylmethacrylate. Clin. Orthop. Relat. Res., v.188, p.303-308, 1984.

WILTSE, M.D.; HALL, R.H.; STENEHJEM, J.C. Experimental studies regarding the possible use of self-curing acrylic in orthopaedic surgery. J. Bone J. Surg. Am., v.39, p.961-972, 1957.

YOUNG, W. Secondary injury mechanisms in acute spinal cord injury. J. Emerg. Med., v.11, p.13-22, 1993. 